\title{
Cardiac tamponade in a case of meningococcal meningitis
}

\author{
PAUL GUERET, MAURICE BUCHALTER \\ From the Cardiology Department, Royal City of Dublin Hospital, Dublin, Ireland
}

SUMMARY A 17 year old girl developed cardiac tamponade during the course of a meningococcal meningeal illness. Pericardiotomy was eventually required to alleviate the tamponade.

\section{Case report}

The patient, a 17 year old girl, presented to another institution with a 36 hour history of headache and vomiting. On admission she was pyrexial and had pronounced photophobia and neck stiffness. Cardiac auscultation was normal. The clinical diagnosis of meningitis was confirmed when a lumbar puncture yielded cloudy cerebrospinal fluid containing no glucose, protein $>200 \mathrm{mg} / \mathrm{dl}$, white cell count $>12000$ cells $/ \mathrm{ml}$. Staining showed gram negative intracellular diplococci, and culture confirmed the growth of Neisseria meningitidis.

Treatment with intravenous benzyl penicillin, one megaunit hourly, was started. The next day she became tachypnoeic and later complained of pleuritic chest pain. Auscultation showed a pleuropericardial rub. Electrocardiogram showed changes consistent with pericarditis. Echocardiography one day later showed a moderately large pericardial effusion without evidence of tamponade. White cell count was $14300 \times 10^{9} / 1$ of which $90 \%$ were polymorphs. She was transferred to our unit for specialist care.

Forty eight hours after transfer her condition deteriorated and pronounced respiratory distress developed. Echocardiography showed enlargement of the pericardial effusion with tamponade, as demonstrated by right ventricular outflow tract collapse during diastole.

Pericardiocentesis produced $200 \mathrm{ml}$ of turbid straw coloured fluid, containing abundant white cells, $50 \%$ of which were polymorphs and $50 \%$ lymphocytes. The fluid contained $100 \mathrm{mg} / \mathrm{dl}$ of protein. Gram stain and culture were negative. The patient improved considerably but only transiently. Thirty six hours later echocardiography again showed tam-

Requests for reprints to Dr Paul Guéret, Cardiology Department, Royal City of Dublin Hospital, Baggot Street, Dublin 4, Ireland. ponade, but on this occasion a large solid element could be seen impinging on the right ventricle (Figure). This was presumed to be thrombus secondary to aspiration trauma. The patient's condition deteriorated and white cell count was $23000 \times$ $10^{9} / 1$.

A further pericardiocentesis was attempted, but it was impossible to aspirate sufficient fluid to alleviate the tamponade. In consultation with the cardiac surgeon pericardiotomy was deemed to be necessary.

At operation several very large fibrin clots were noted, compressing the right and left ventricles. More than $400 \mathrm{ml}$ of sterile cloudy fluid was removed from a very fibrinous pericardial sac. The volume of the fibrin clots was estimated to be $>100 \mathrm{ml}$.

Postoperative recovery was uneventful and the patient was discharged two weeks later. There was no further complication at a follow up at four months.

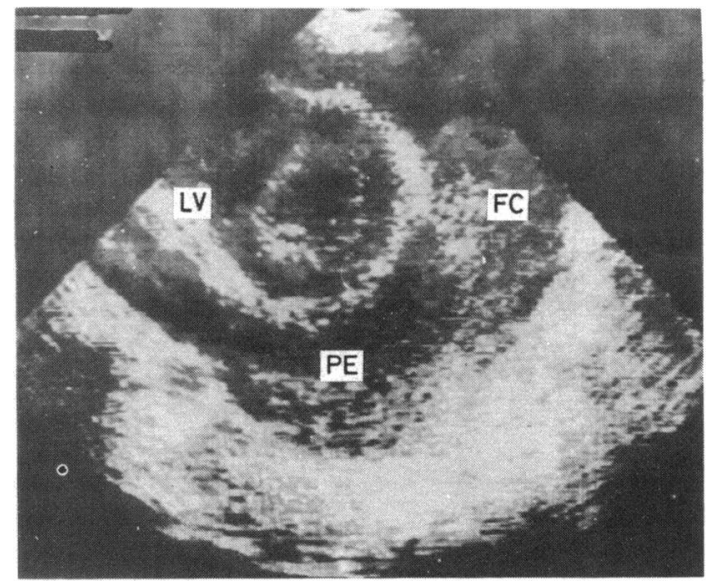

Figure Echocardiograph in parasternal short axis view showing large fibrin clot in pericardial effusion. $L V$, left ventricle; PE, pericardial effusion; FC, fibrin clot. 


\section{Discussion}

Pericarditis complicating meningococcal infection has been well described. The reported incidence varies from $<1 \%,{ }^{12}$ to $4-6 \%,{ }^{3}$ to as much as $19 \%,{ }^{45}$ depending on the various criteria used. Cardiac tamponade as a complication of primary meningococcal pericardial infection is also well described, but review of published reports showed only five cases of tamponade caused by primary meningeal infection. 3467

The effusion is commonly believed to be secondary to bacterial seeding of the pericardium. Herrick in 1918 who described 12 cases of pericarditis secondary to meningococcal meningitis found at least two cases with positive gram stain in the pericardial aspirate; indeed one of these cases had tamponade as well. ${ }^{3}$ Morse $e t$ al, however, believe that the effusion is likely to be secondary to an immunological hypersensitivity reaction. ${ }^{4}$

In our case, in view of the rapid onset of the effusion, the large number of white cells in the aspirate, and the fibrinous nature of the effusion and pericardium, we feel that bacterial seeding is likely to be the cause of the effusion.

Of the five previously described cases, one was not diagnosed until after death, ${ }^{3}$ the others were all treated by pericardiocentesis, and two cases were also given steroids. ${ }^{67}$ The presence of fibrin clots and the failure of pericardiocentesis in our case led us to surgery. We believe this to be the first reported case of tamponade secondary to meningeal meningococcal infection that has required pericardiotomy.

\section{References}

1 Johnson M, Alvin A. A 12 year review of acute bacterial meningitis in Stockholm. Scand F Infect Dis 1971; 3: 141-9.

2 Hoyne AL, Browne RH. 727 meningococcic cases, an analysis. Ann Intern Med 1948; 28: 24-32.

3 Herrick WW. Meningococcic pericarditis with a report of 12 cases. Med Clin North Am 1918; 2: 411-26.

4 Morse JR, Ortesky MI, Hudson JA. Pericarditis as a complication of meningococcal pericarditis. Ann Intern Med 1971; 74: 212-7.

5 Laird WP, Nelson JD, Huffines FD. The frequency of pericardial effusion in bacterial meningitis. Pediatrics 1979; 63: 764-70.

6 Lukash WM. Massive pericardial effusion due to meningococcal meningitis. $\mathcal{F} A M A$ 1963; 185: 598-600.

7 Lebowitz WB, Nespole AJ. Purulent pericarditis complicating meningococcal meningitis. Am $\mathcal{f}$ Dis Child 1967; 113: 385-9. 